\title{
Efficient Modeling of Welding Mechanics Using Mesh Superposition*
}

\author{
by IKUSHIMA Kazuki**, ASHIDA Ryo *** and SHIBAHARA Masakazu**
}

\begin{abstract}
Thermal elastic plastic analysis is used to predict welding deformation and residual stress. In the thermal elastic plastic analysis, the non-linear mechanical behavior of a welded is part consecutively analyzed. Therefore, to evaluate a complex structure, this analysis requires detailed modeling and a huge amount of computing time. However, the welded part is generally small compared to the whole structure, and it is very difficult to construct an analysis model for the whole structure that considers the welded part. In this research, the mesh superposition method is introduced to thermal elastic plastic analysis. The mesh superposition method makes it is possible to construct separate analysis models for the whole structure and the welded part. The applicability of the proposed method was investigated by comparison with a conventional analysis method. The result showed that the proposed analysis method has almost the same accuracy as the conventional method. The proposed method was also applied to the analysis of welding deformation in a structure consisting of multiple parts. The result indicated that the proposed method can effectively construct the complex analysis model by using a local mesh to join the multiple parts in a global mesh.
\end{abstract}

Key Words: Mesh superposition, Finite element method, Thermal elastic plastic analysis, Welding deformation, Residual stress

\section{Introduction}

In the construction of structures such as ships, buildings, and bridges, welding is widely utilized to join the structural members. This may cause deformation and residual stress in the welded part, leading to various problems. Thus, these factors need to be evaluated in advance of construction.

Welding deformation and residual stress can be predicted using nonlinear thermal elastic plastic finite element analysis (TEPFEA $)^{1}$. However, TEP-FEA requires huge computing resources. In addition, TEP-FEA includes strong nonlinearity near the weld torch, and this demands very detailed modeling near the welded part. Therefore, TEP-FEA has limited analytical scope, and large labor costs are necessary to construct a model for TEP-FEA.

To achieve an efficient analysis in TEP-FEA, various methods have been developed, such as the dynamic mesh refinement method (DMRM) ${ }^{2}$, 3). In the DMRM, mesh is adaptively refined according to the movement of weld torch and the cost for modeling is reduced. The author has also proposed a new analysis method called the idealized explicit finite element method (IEFEM) ${ }^{4)}$. The IEFEM is based on the dynamic explicit FEM $^{5)}$, and its efficiency was increased for welding problems. Although the accuracy of the IEFEM is almost the same as the conventional method, the IEFEM is very fast and saves memory.

As an alternative approach, the mesh superposition method has been proposed for efficient modeling in structural analysis ${ }^{4}$. In this method, local detailed meshes are superposed onto the region of

* Received: 2019.11.21, Presented at Visual-JW or WSE 2019

** Member, Graduate School of Engineering, Osaka Prefecture University

*** Student Member, Graduate School of Engineering, Osaka Prefecture University interest in a global mesh. Using this procedure, mesh superposition can achieve an accurate analysis with efficient modeling. For welding analysis, the mesh superposition method can be effectively applied to the nonlinear region near a welded part because a nonlinear region concentrates near the weld. However, in this method, complex procedures are performed to superpose meshes, and this increases the computing time.

In this study, to achieve efficient modeling, the mesh superposition method was introduced to the IEFEM. To validate the accuracy of the proposed method, it was compared with a conventional method. In addition, to show the usefulness of the proposed method, it was applied to the analysis of a welding assembly problem.

\section{Formulation of TEP-FEA with mesh superposition method}

\subsection{Heat conduction analysis}

In the TEP-FEA, unsteady heat conduction analysis is performed to obtain the thermal history. Defining the weight function as $T^{*}$ and applying the weighted residual method to the governing equation of unsteady heat conduction analysis yields the following equation:

$$
\int_{\Omega} T^{*}\left(c \rho \frac{\partial T}{\partial t}-\lambda \frac{\partial^{2} T}{\partial x_{i}^{2}}\right) d \Omega=\int_{\Omega} T^{*} q d \Omega
$$

where $c, \rho, \lambda, \alpha_{c}, T, T_{c}$, and $q$ are thermal capacity, density, thermal conductivity, heat transfer coefficient, temperature, room temperature and heat density, respectively; $\Omega$ is the analysis domain; and $\Gamma$ is heat transfer surface.

In the mesh superposition method, the detailed (local) mesh is superposed onto the mesh for the whole analysis domain $\Omega$ (global mesh), as shown in Fig. 1. The domains where the local 


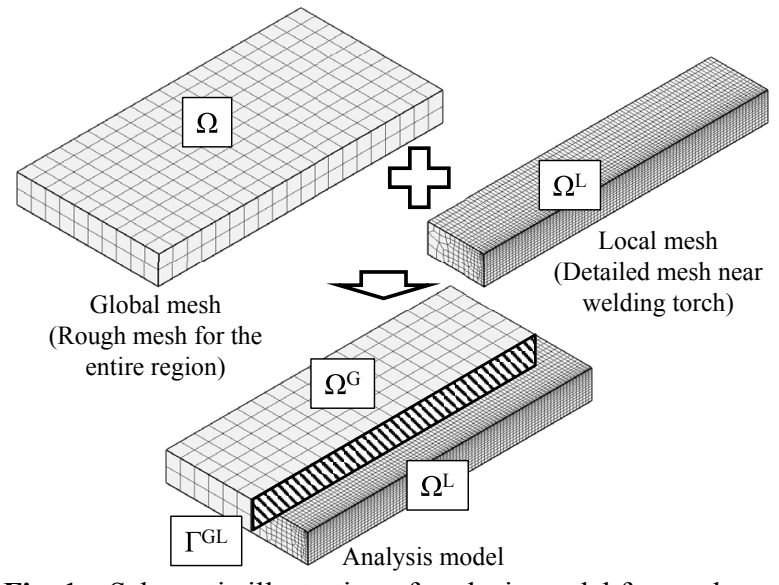

Fig. 1 Schematic illustration of analysis model for mesh superposition method.

mesh is placed and only the global mesh exists are defined as $\Omega^{L}$ and $\Omega^{G}\left(=\Omega / \Omega^{L}\right)$, respectively. The boundary between the domains $\Omega^{G}$ and $\Omega^{L}$ is defined as $\Gamma^{G L}$. The formulation for the region $\Omega^{G}$ is same as the ordinary FEM. The temperatures in domain $\Omega^{L}$ is given by Eq. (2). On the boundary $\Gamma^{G L}$, the temperature is fixed as in Eq. (3) to preserve the continuity of the temperature field between the global mesh and the local mesh.

$$
\begin{aligned}
& T=\boldsymbol{N}^{G} \boldsymbol{T}^{G n}+\boldsymbol{N}^{L} \boldsymbol{T}^{L n} \text { in } \Omega^{L} \\
& \boldsymbol{T}^{L n}=\{0\} \quad \text { on } \Gamma^{G L}
\end{aligned}
$$

where $\boldsymbol{N}^{G}, \boldsymbol{N}^{L}, \boldsymbol{T}^{G n}$, and $\boldsymbol{T}^{L n}$ are the shape function and nodal temperature for the global and local mesh, respectively. As shown in Eq. (2), the temperature field is approximated in $\Omega^{L}$ by superposing the approximate function of the local mesh to that of the global mesh. In the same way, the weighting function is approximated as:

$$
T^{*}=\boldsymbol{N}^{G} \boldsymbol{T}^{* G n}+\boldsymbol{N}^{L} \boldsymbol{T}^{* L n} \text { in } \Omega^{L}
$$

Substituting Eqs. (2)-(4) into Eq. (1) and considering boundary condition yields:

$$
\begin{aligned}
{\left[\begin{array}{ll}
\boldsymbol{C}^{G G} & \boldsymbol{C}^{G L} \\
\boldsymbol{C}^{L G} & \boldsymbol{C}^{L L}
\end{array}\right] \frac{\partial}{\partial t}\left\{\begin{array}{l}
\boldsymbol{T}^{G n} \\
\boldsymbol{T}^{L n}
\end{array}\right\}+\left[\begin{array}{ll}
\boldsymbol{K}^{G G} & \boldsymbol{K}^{G L} \\
\boldsymbol{K}^{L G} & \boldsymbol{K}^{L L}
\end{array}\right]\left\{\begin{array}{l}
\boldsymbol{T}^{G n} \\
\boldsymbol{T}^{L n}
\end{array}\right\} } \\
=\left[\begin{array}{ll}
\boldsymbol{A}^{G G} & \boldsymbol{A}^{G L} \\
\boldsymbol{A}^{L G} & \boldsymbol{A}^{L L}
\end{array}\right]\left\{\begin{array}{l}
\boldsymbol{T}^{G n} \\
\boldsymbol{T}^{L n}
\end{array}\right\}+\left\{\begin{array}{l}
\boldsymbol{Q}^{G} \\
\boldsymbol{Q}^{L}
\end{array}\right\}
\end{aligned}
$$

where $\boldsymbol{C}^{a b}, \boldsymbol{K}^{a b}, \boldsymbol{A}^{a b}$, and $\boldsymbol{Q}^{a}$ are the heat capacity matrix, heat conduction matrix, heat transfer matrix, and heat vector, respectively. $a$ and $b$ in Eq. (5) denote either region $G$ or $L$. These matrices and the vector are determined by the following integrations:

$$
\begin{aligned}
& \boldsymbol{C}^{a b}=\int_{\Omega^{L}} c \rho \boldsymbol{N}^{a^{T}} \boldsymbol{N}^{b} d \Omega \\
& \boldsymbol{K}^{a b}=\int_{\Omega^{L}} \lambda \frac{\partial \boldsymbol{N}^{a^{T}}}{\partial x_{i}} \frac{\partial \boldsymbol{N}^{b}}{\partial x_{i}} d \Omega \\
& \boldsymbol{A}^{a b}=\int_{\Gamma^{L}}-\alpha_{c} \boldsymbol{N}^{a^{T}} \boldsymbol{N}^{b} d \Gamma \\
& \boldsymbol{Q}^{a}=\int_{\Omega^{L}} q \boldsymbol{N}^{a^{T}} d \Omega+\int_{\Gamma^{L}} \alpha_{c} T_{c} \boldsymbol{N}^{a^{T}} d \Gamma
\end{aligned}
$$

The change of temperature during heating is steep while that during cooling is moderate. With these equations, the time domain is implicitly discretized because a wide range of time increments can be used in an implicit form. Here, the Crank-Nicolson method is employed. The integrations in Eqs. (6)-(9) are performed using Gauss integration, for which the integration, shape function, and its derivative in the global mesh are necessary. In the developed method, the location of the Gauss integration point for the local mesh in the coordinate system of the global mesh is determined before the analysis ${ }^{6}$. The determined location is referenced during the integration to calculate the shape function and its derivatives in global mesh.

\subsection{Thermal elastic plastic analysis}

In the TEP analysis, the displacement increment is determined at each time step by referring to the temperature field history obtained by the heat conduction analysis. The virtual work principal is given in Eq. (10). In this equation, external forces are not considered.

$$
\int_{\Omega} \delta \boldsymbol{\varepsilon}^{T}(\boldsymbol{\sigma}+\Delta \boldsymbol{\sigma}) d \Omega=0
$$

Here, $\delta \boldsymbol{\varepsilon}, \boldsymbol{\sigma}$, and $\Delta \boldsymbol{\sigma}$ are the virtual strain, stress, and stress increment, respectively. The formulation for the region $\Omega^{G}$ is same as the ordinary FEM. Increments of displacement and strain in the local region $\Omega^{L}$ are defined as follows:

$$
\begin{aligned}
& \Delta \boldsymbol{u}=\boldsymbol{N}^{G} \Delta \boldsymbol{u}^{G n}+\boldsymbol{N}^{L} \Delta \boldsymbol{u}^{L n} \quad \text { in } \Omega^{L} \\
& \Delta \boldsymbol{u}^{L n}=\{0\} \quad \text { on } \Gamma^{L G} \\
& \Delta \boldsymbol{\varepsilon}=\boldsymbol{B}^{G} \Delta \boldsymbol{u}^{G n}+\boldsymbol{B}^{L} \Delta \boldsymbol{u}^{L n} \text { in } \Omega^{L}
\end{aligned}
$$

where $\boldsymbol{B}^{G}, \boldsymbol{B}^{L}, \Delta \boldsymbol{u}^{G n}$, and $\Delta \boldsymbol{u}^{L n}$ are the displacement-strain relation matrix and nodal displacement increment for the global and local meshes, respectively.

The virtual strain $\delta \boldsymbol{\varepsilon}$ is defined using the nodal virtual displacement in the global mesh $\delta \boldsymbol{u}^{G n}$ and local mesh $\delta \boldsymbol{u}^{L n}$ as follows:

$$
\delta \boldsymbol{\varepsilon}=\boldsymbol{B}^{G} \delta \boldsymbol{u}^{G n}+\boldsymbol{B}^{L} \delta \boldsymbol{u}^{L n} \quad \text { in } \Omega^{L}
$$

The stress increment $\Delta \boldsymbol{\sigma}$ is defined as follows:

$$
\Delta \boldsymbol{\sigma}=\boldsymbol{D}^{L}\left(\Delta \boldsymbol{\varepsilon}-\Delta \boldsymbol{\varepsilon}_{T}^{L}-\Delta \boldsymbol{\varepsilon}_{p}^{L}\right) \text { in } \Omega^{L}
$$

where $\boldsymbol{D}^{L}$ are the stress-strain relation matrix in the local meshes; $\Delta \boldsymbol{\varepsilon}_{T}^{L}$ are the thermal strain increments in the local meshes; and $\Delta \boldsymbol{\varepsilon}_{p}^{L}$ is the plastic strain increment in the local mesh. Nonlinearity due to plastic deformation is assumed only in $\Omega^{L}$. Elastic strain and thermal strain are considered in $\Omega^{G}$.

Substituting Eqs. (11)-(15) into Eq. (10) yields the following equation:

$$
\left[\begin{array}{ll}
\boldsymbol{K}^{G G} & \boldsymbol{K}^{G L} \\
\boldsymbol{K}^{L G} & \boldsymbol{K}^{L L}
\end{array}\right]\left\{\begin{array}{c}
\Delta \boldsymbol{u}^{G n} \\
\Delta \boldsymbol{u}^{L n}
\end{array}\right\}=\left\{\begin{array}{c}
\Delta \boldsymbol{F}^{G} \\
\Delta \boldsymbol{F}^{L}
\end{array}\right\}-\left\{\begin{array}{c}
\boldsymbol{f}^{G} \\
\boldsymbol{f}^{L}
\end{array}\right\}
$$

Each term in Eq. (16) is determined by the following integrations: 


$$
\begin{aligned}
& \boldsymbol{K}^{a b}=\int_{\Omega^{L}} \boldsymbol{B}^{a^{T}} \boldsymbol{D}^{L} \boldsymbol{B}^{b} d \Omega \\
& \boldsymbol{F}^{a}=\int_{\Omega^{L}} \boldsymbol{B}^{a^{T}} \boldsymbol{D}^{L} \Delta \boldsymbol{\varepsilon}_{T}^{L} d \Omega \\
& \boldsymbol{f}^{a}=\int_{\Omega^{L}} \boldsymbol{B}^{a^{T}} \boldsymbol{\sigma}^{L} d \Omega
\end{aligned}
$$

The obtained stiffness equation is iteratively solved until the whole system reaches a static equilibrium state.

\subsection{Introduction to IEFEM}

TEP-FEA can be conducted by solving Eqs. (5) and (16) for each time step. However, the non-zero pattern of the stiffness matrix in the mesh superposition method is as shown in Fig. 2 and is different from that for the conventional method. This leads to an increased amount of fill-in when solving these equations using a direct solver and may increase the computing time and memory consumption. To avoid this problem, the IEFEM was adopted for efficient computation.

Application of the IEFEM for heat conduction and thermal elastic plastic analyses is described in detail in the literature ${ }^{4,7)}$. In the IEFEM, it is necessary to integrate the right-hand side (RHS) of Eq. (16) until convergence is obtained. The integration contains complex procedures that reference the displacement-strain matrix in the global mesh. This may increase the computation burden and make it inefficient.

For efficient computation, as shown in Fig. 3, the following procedure was employed in the developed method. First, the mass matrix and damping matrix are determined. The stiffness matrix is also calculated and stored in the compressed row storage format to reduce memory consumption. Next, the displacement vector is calculated using the dynamic explicit FEM. After that, the residual force vector is calculated by multiplying the stiffness matrix and the obtained displacement vector instead of integrating the RHS of Eq. (16). After iterating these steps $\mathrm{N}$ times (as determined by the user), an accurate residual force vector is calculated by integrating the RHS of Eq. (16). The convergence is evaluated based on this accurate residual force vector. The analysis proceeds to the next time step when convergence is obtained while the above steps are performed again using the accurate residual force vector as the load

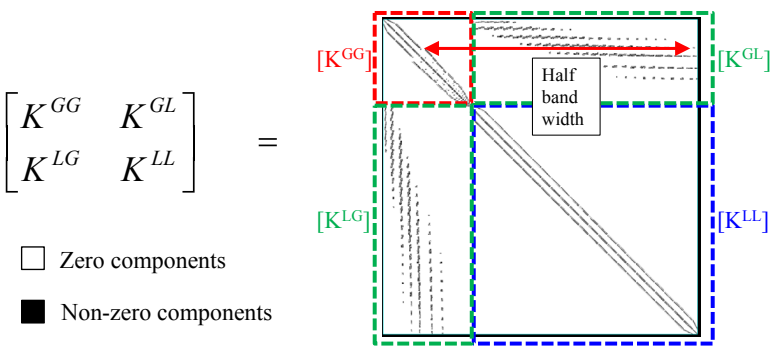

Fig. 2 Schematic illustration of non-zero structure of stiffness matrix in mesh superposition method. vector when convergence is not obtained. This procedure reduces the need for complex integration. The same procedure is also employed in the heat conduction analysis [Eq. (5)].

As shown in Fig, 4, the above analysis system makes it possible to superpose the detailed local mesh for the welded part onto the relatively coarse global mesh for the whole structure. The local mesh is selected according to the type of weld joint, and detailed mesh divisions are adopted. In this method, complex mesh divisions for the welded part are not necessary in the global mesh. Thus, the construction of the analysis model has increased flexibility.

\section{Validation of proposed method}

The proposed method was applied to an analysis of the fundamental bead-on-plate problem shown in Fig. 5. The dimensions of the analysis model were $100 \mathrm{~mm}$ in length, $50 \mathrm{~mm}$ in width, and $10 \mathrm{~mm}$ in thickness. Due to the mechanical symmetry, half of the plate was modeled in the $y$-direction (width). The welding conditions were a current of $120 \mathrm{~A}$, a voltage of $10 \mathrm{~V}$,

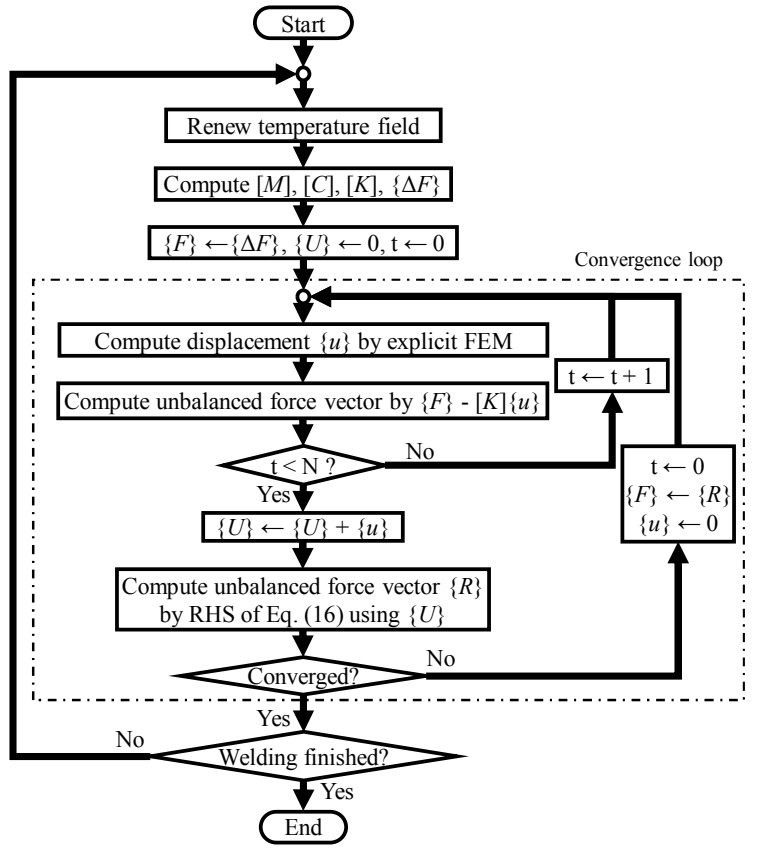

Fig. 3 Logical flow of IEFEM employing mesh superposition.

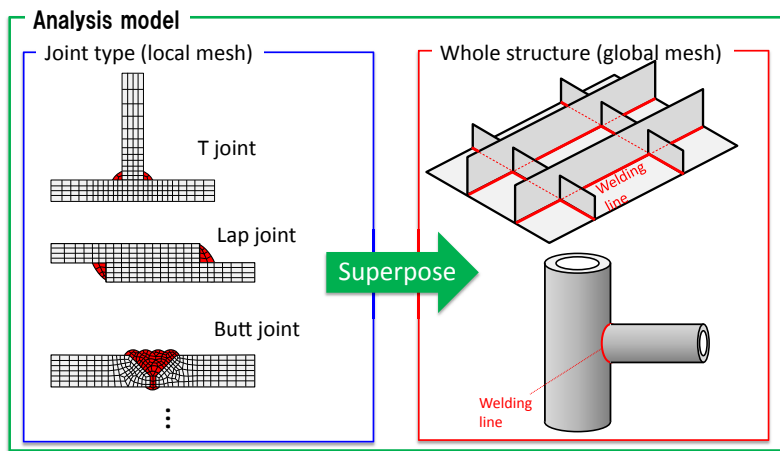

Fig. 4 Concept of analysis model in mesh superposition method. 


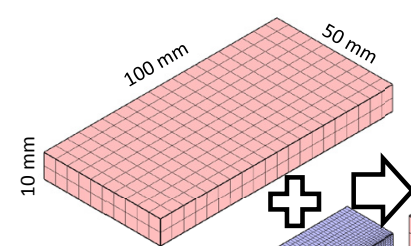

(a) Global mesh.

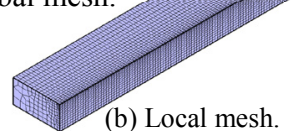

Fig. 5 Bead-on-plate analysis model.

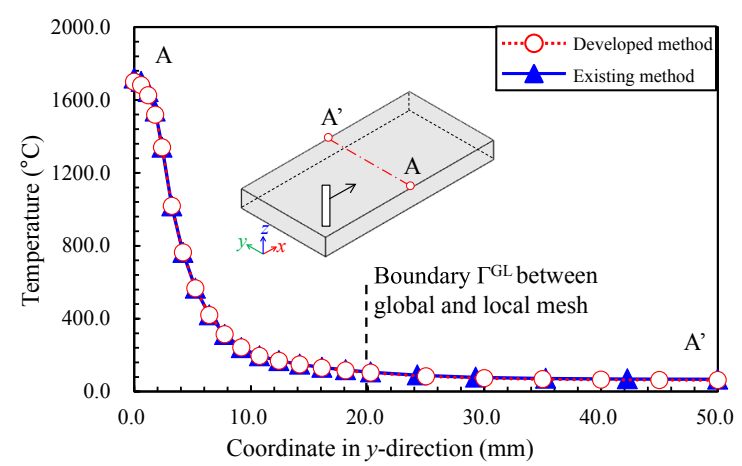

Fig. 6 Comparison of maximum temperature distribution along line A-A'.

speed of $4.0 \mathrm{~mm} / \mathrm{s}$, and an efficiency of 0.5 . The plate material was assumed to be mild steel ${ }^{4}$. The mesh divisions used in this analysis are shown in Fig. 5. Figure 5(a) shows divisions for the global mesh, where a relatively coarse mesh division was adopted with 400 elements. Figure 5(b) shows the mesh divisions for the local mesh. To analyze the nonlinear behavior, detailed mesh division was adopted in the local mesh and the number of elements was 10,620 . In this analysis, the local mesh was generated so that more than 2 elements in the local mesh are included in an element in global mesh. As shown here, we can construct global and local meshes separately in the proposed method. It can be expected that the cost in the modeling is reduced by this characteristic, especially in the analysis of complex structures. For comparison, analysis using an implicit FEM solver was conducted. In the implicit FEM solver, the skyline method was employed to solve the simultaneous equations. Regarding the number of iterations in Fig. 3, N=200 was employed.

Figure 6 shows the maximum temperature distribution along line A-A' in Fig. 5. The maximum temperature distribution for the proposed method quantitatively agrees with that for the conventional method. In particular, a continuous distribution was obtained on the boundary between the global and local mesh.

Figures 7 and 8 show the distributions of the transverse shrinkage and the angular distortion, respectively. These figures show that a typical welding deformation was obtained by both the conventional and proposed methods and that the deformation produced by each method is similar. Figure 9 shows the distribution of residual stress in the $x$-direction $\left(\sigma_{\mathrm{x}}\right)$. The figure

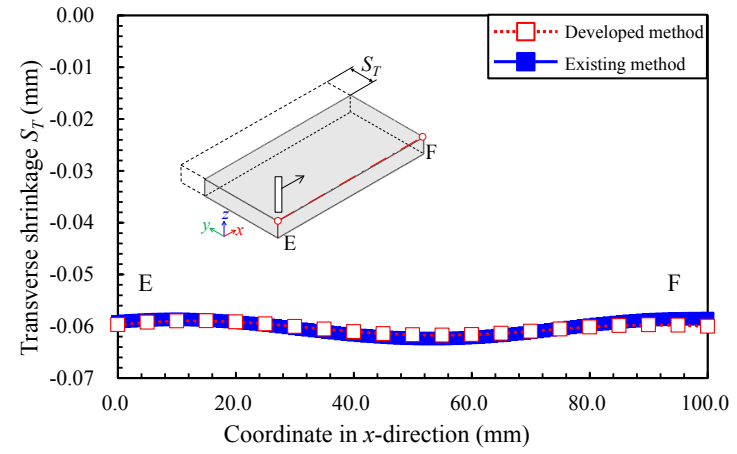

Fig. 7 Comparison of transverse shrinkage distribution.

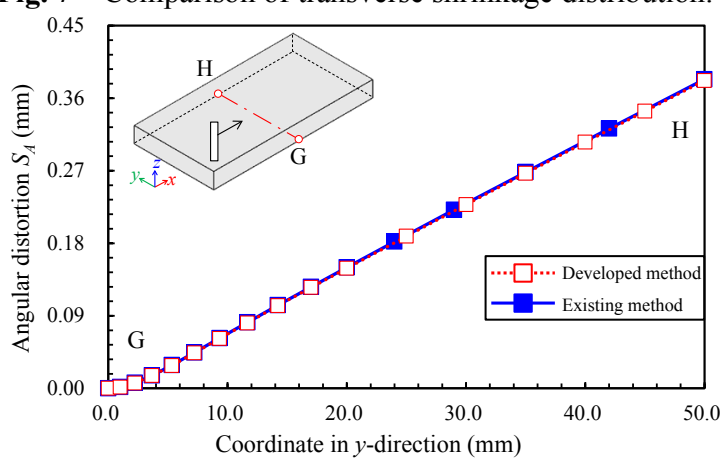

Fig. 8 Comparison of angular distortion distribution.

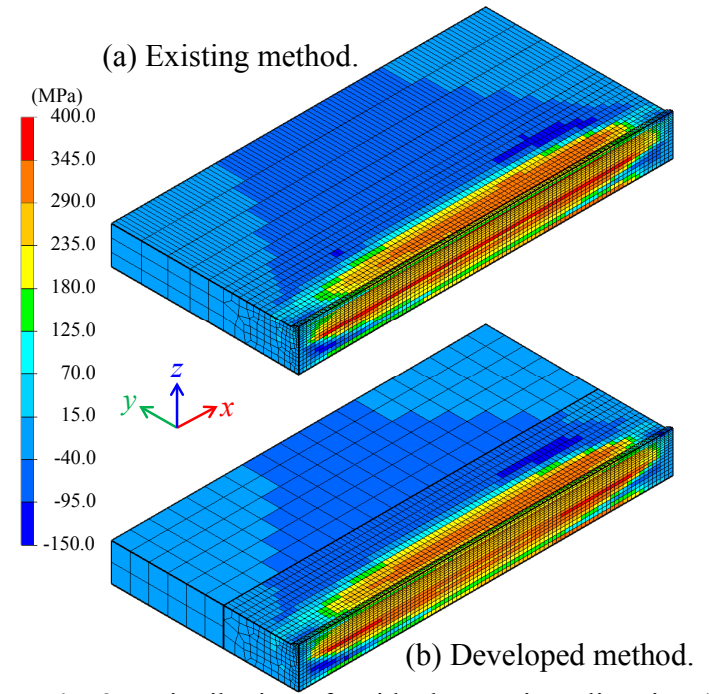

Fig. 9 Distribution of residual stress in $x$-direction $\left(\sigma_{x}\right)$. shows that the residual stress distribution obtained by the proposed method agrees very well with that obtained by the conventional method. In addition, it can be seen that the residual stress distribution obtained by the proposed method is continuous across the boundary between domains $\Omega^{G}$ and $\Omega^{L}$.

The above results indicate that the proposed method can analyze welding mechanical problems with the same accuracy as the conventional method.

\section{Application to complex structure}

To demonstrate the applicability and usefulness of the proposed method for the analysis of complex structures, it was applied to the 


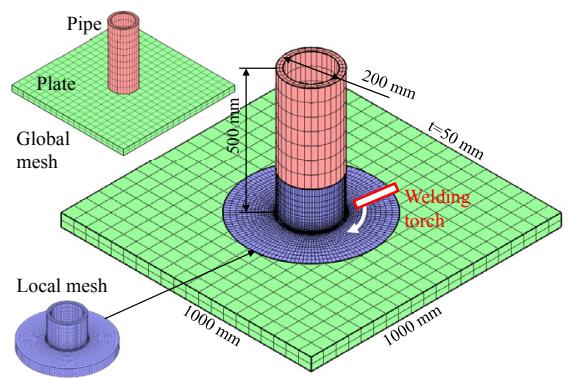

(a) Whole model.

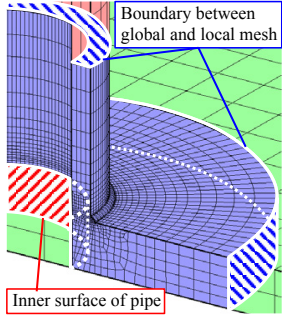

(b) Zoomed view.

Fig. 10 Welding assembly analysis model.

welding mechanical analysis shown in Fig. 10. The number of elements and nodes in the global mesh were 1,440 and 2,379, respectively. Those in the local mesh were 47,872 and 41,984 , respectively. In this model, the pipe and plate were separately modeled in the global mesh as shown in Fig. 10(a). The welded part was modeled in the local mesh. By superposing the local mesh onto the global mesh, multiple parts were joined. The nodes in the global mesh were not shared at all between the pipe and plate, that is, they were completely separate parts. In the local mesh, as shown in Fig. 10(b), the displacements at the boundaries between the plate and local mesh and between the pipe and local mesh were fixed to preserve the continuity of the displacement among the local mesh, pipe, and plate. The pipe pierced the plate and this was modeled by not constraining the displacement on the inner surface of the local mesh. The material of the pipe and plate was the same mild steel described in the previous chapter.

Figure 11 shows the distribution of displacement in the $x$ direction after complete cooling. A shrinking deformation perpendicular to the welding bead can be seen. Although the mesh division is different on either side of the boundary between the local and global meshes, a continuous displacement distribution is obtained. This is due to the constraint between the local and global meshes. In the same way, Fig. 12 shows the distribution of displacement in the $z$-direction. It can be seen that the surrounding part rises toward the central part due to the angular distortion induced by the weld. A continuous distribution of displacement in the $z$-direction is obtained.

As shown by the above discussion, analysis of a complex structure can easily be performed using the mesh superposition method by superposing the global mesh for each part and the local mesh for the welded part. This can drastically reduce the complexity of constructing analysis models.

\section{Conclusions}

This study achieved an advanced analysis method for welding mechanics problems by introducing the mesh superposition

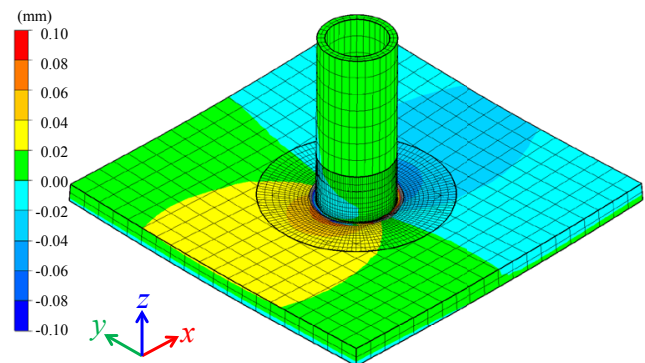

Fig. 11 Distribution of displacement in x-direction.

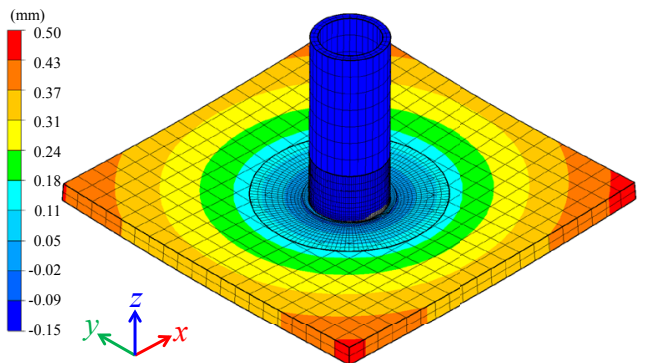

Fig. 12 Distribution of displacement in z-direction.

method to thermal elastic plastic analysis and heat conduction analysis. In addition, to achieve efficient computation, the IEFEM was employed. The proposed method was applied to a fundamental bead-on-plate problem to investigate the analysis accuracy. As a result, it was shown that the results obtained by the proposed method have almost the same accuracy as those obtained by the conventional method. The proposed method was also applied to the analysis of a welded pipe and plate assembly to show its applicability and usefulness. As a result, it was indicated that the proposed method is effective for the analysis of complex structures consisting of multiple parts.

\section{Reference}

1) Y. Ueda and T. Yamakawa: Analysis of Thermal Elastic-Plastic Behavior of Metals during Welding by Finite Element Method, J. JWS, 42-6(1973), 567-577.

2) H. Huang, H. Murakawa: Dynamic mesh refinement and iterative substructure method for fillet welding thermo-mechanical analysis, Comput. Model. Eng. Sci., 106-3(2015), 187-201.

3) H. Huang, H. Serizawa, J. Wang, H. Murakawa: Development of Thermal Elastic Elastic-plastic FEM for Line Heating with Remeshing Technique, Quart. J. JWS, 31-4(2013), 134s-137s.

4) M. Shibahara, K. Ikushima, S. Itoh and K. Masaoka: Computational Method for Transient Welding Deformation and Stress for Large Scale Structure Based on Dynamic Explicit FEM, Quart. J. JWS, 29-1(2011), 1-9.

5) G. Yagawa: Structural engineering handbook, Maruzen (2004).

6) S. Nakasumi, K. Suzuki, H. Ohtsubo and D. Fujii: Mixed analysis of shell and solid elements using overlaying mesh method, J. Soc. Naval Archit. Jpn., 189(2001), 219-224.

7) K. Ikushima, S. Itoh and M. Shibahara: Heat Conduction Analysis of Welding Using Idealized Explicit FEM, Quart. J. JWS, 31-4 (2013), 153s-157s. 